\title{
Genetics of Aggregation Pattern Mutations in the Cellular Slime Mould Dictyostelium discoideum
}

\author{
By FIONA M. ROSS AND PETER C. NEWELL \\ Department of Biochemistry, University of Oxford, \\ South Parks Road, Oxford OX1 $3 Q U$
}

(Received 3 February 1979)

\begin{abstract}
A class of aggregation pattern mutants called 'streamers' have been isolated from Dictyostelium discoideum and analysed genetically. The streamer phenotype is the formation of very large streams of centripetally moving amoebae which are collected from abnormally large territories during the aggregation phase of this organism. Such mutants do not show the pleiotropic developmental defects seen with most other classes of aggregation mutants and after the abnormal aggregation phase they develop into normally differentiated stalk cells and spores. Twenty-four haploid streamers were isolated and assigned to seven complementation groups, $\operatorname{stm} A$ to $\operatorname{stm} G$, after selecting diploids formed between pairs of the mutants. The complementation loci were assigned to the following linkage groups using parasexual genetic techniques: $\operatorname{stm} A$ and $\operatorname{stm} F$, linkage group VII; $\operatorname{stm} B, \operatorname{stm} D$ and $\operatorname{stm} G$, linkage group II; $\operatorname{stm} C$ and $\operatorname{stm} E$, linkage group III. Use was made of a new temperature sensitive for growth marker, $t s g K 21$, which was assigned to linkage group VII. The total number of complementation groups giving the streamer phenotype is estimated from statistical calculation, based on the frequency of allelism, to be between seven and nine.
\end{abstract}

\section{INTRODUCTION}

The cellular slime moulds are currently exciting considerable attention from biologists because of their relatively simple programme of differentiation that is triggered by starvation (reviewed by Loomis, 1975) and because of their unusual aggregative phase during which cell communication occurs over large distances by means of a pulsatile chemical signalling system (reviewed by Newell, 1977).

One approach to the study of such a system is to isolate and study developmental mutants. Unfortunately, as for other systems (such as Bacillus sporulation; Mandelstam, 1969), the process of development is sequential such that the absence of a particular developmental event usually blocks the normal appearance of all subsequent events. As a consequence, such developmental mutants have pleiotropic defects that are tantalizingly difficult to analyse. It seems unlikely, however, that all enzymes or other proteins formed during development act as inducers whether the programme is a simple linear one as proposed by Loomis et al. (1976) or a complex causal net as proposed by Francis (1977). Mutants that affect the pattern of aggregation or later development without causing pleiotropic defects might possess mutations in genes concerned with events on branches off the main regulation sequence. In this class one might expect to find, for example, mutations in those aggregation functions that regulate variables such as the frequency of centre formation, the dimensions of aggregation territories and streams, and the timing and synchronization of aggregational and developmental events. 
For this study we chose those mutants that showed abnormally large aggregation streams (hence the name 'streamers') that clearly lacked the normal regulatory system that limited the range of the chemotactic signalling system.

This report describes a genetic study of 24 streamer mutants of Dictyostelium discoideum undertaken to ascertain the number of complementation groups that are concerned with this phenotype and to assign them to linkage groups prior to a detailed biochemical investigation. The parasexual genetic techniques employed have recently been reviewed (Newell, 1978).

\section{METHODS}

Media and chemicals. The composition and methods of preparation of nutrient (SM) and selective media, salts solution (SS) and the chemicals used were as described previously (Mosses et al., 1975; Ratner $\&$ Newell, 1978). For the selective agar plates containing $\mathrm{CoCl}_{2}$ it was found that a frequently encountered problem of uneven lawns of bacteria could be circumvented by the simple expedient of allowing the $\mathrm{CoCl}_{2}$ to remain in the autoclaved medium for $15 \mathrm{~min}$ before stirring and pouring. Agar plates containing $p$-fluorophenylalanine (PFP) were made by adding $0.1 \%$ (w/v) PFP to SM agar before pouring. $\mathrm{KK}_{2}$ plates were prepared as described by Green \& Newell (1975). Dextran (clinical grade) and PFP were obtained from Sigma, $p$-nitrophenyl- $\alpha$-D-mannopyranoside (A grade) from Calbiochem, and $N$-methyl- $N$-nitro- $N$-nitrosoguanidine (NTG) from Ralph Emanuel, Wembley.

Strains. Dictyostelium discoideum stocks were grown on lawns of the cobalt-resistant mutant of Klebsiella aerogenes designated strain OXF1 (Williams \& Newell, 1976). All D. discoideum strains used are derivatives of NC4 (Raper, 1935). The nomenclature system used is based on that of Demerec et al. (1966) (see Kessin et al., 1974). Table 1 shows the origins and genotypes of all haploid strains used. Phenotypes, when given in subsequent tables and in the text, are denoted by the locus symbol with an initial capital (in roman type), the wild type being designated by the superscript + .

Maintenance of stocks. Standard procedures (Williams et al., 1974) were employed for the handling of amoebae and the preservation of spores in silica gel. Some spores were also stored in glycerol as previously described (Ratner \& Newell, 1978). This method is not recommended for periods of more than about 6 months. Mutants in use were maintained in association with $K$. aerogenes by cloning at 7 to $10 \mathrm{~d}$ intervals.

Mutagenesis. Freshly harvested amoebae were incubated at $10^{7} \mathrm{ml}^{-1}$ in SS salts solution for 10 to $45 \mathrm{~min}$ with 200 or $500 \mu \mathrm{g} \mathrm{NTG} \mathrm{ml} l^{-1}$ so as to give approximately $10 \%$ survival. Mutagenized amoebae were washed three times in SS, plated on $\mathrm{KK}_{2}$ plates without a bacterial associate and allowed to develop. The resulting spores (approximately $5 \times 10^{6}$ per plate) were harvested and stored in glycerol at $-20^{\circ} \mathrm{C}$. To screen for mutants, the spores were plated on SM agar at about 50 spores per plate with an inoculum of $K$. aerogenes. Because one division, at most, could have occurred during the development of the spores (Zada-Hames \& Ashworth, 1977), it is very unlikely that any of the mutants obtained using this technique had a common origin. Mutants with a stable streamer phenotype occurred in strain XP55 with an approximate frequency of $5 \times 10^{-4}$ under these conditions.

Formation of diploids. Two haploid strains, one carrying a tsg mutation and the other a bsg mutation, were incubated for 17 to $22 \mathrm{~h}$ as described previously (Newell et al., 1977 a,b). Diploids were formed at a frequency of about $10^{-5}$ and were selected by their ability to grow on a Bacillus subtilis lawn at $27^{\circ} \mathrm{C}$. In all cases two diploids were retained and used in further studies. These are likely to have resulted from independent fusion events.

Segregation of haploids. This was normally done by standard methods (e.g. Williams et al., 1974) using cycloheximide, methanol or $\mathrm{CoCl}_{2}$ as the selective agent. In some cases, where large numbers of cross-over diploids were formed (homozygous for the selective marker) relative to the frequency of haploidization, the diploid was grown on PFP plates at $25.5^{\circ} \mathrm{C}$ for $7 \mathrm{~d}$ before plating on the selective medium (Coukell \& Roxby, 1977). This increased the haploid yield approximately 100 -fold.

Determination of segregant phenotypes. The scoring of most phenotypes followed the methods of Ratner \& Newell (1978) with the following two modifications. Growth on $\mathrm{CoCl}_{2}$-containing plates could be scored at $6 \mathrm{~d}$ (rather than $9 \mathrm{~d}$ ) if even lawns of the bacterial associate were produced by the technique described above. Scoring of growth on plates inoculated with $B$. subtilis was considerably facilitated if the plates spread with the bacteria were incubated for 3 to $6 \mathrm{~h}$ at $37^{\circ} \mathrm{C}$ before transferring the slime mould on to them. This distinguished between haploid and diploid clones by growth rate and virtually eradicated any residual growth of Bsg clones such that accurate scoring could be achieved after $4 \mathrm{~d}$. 


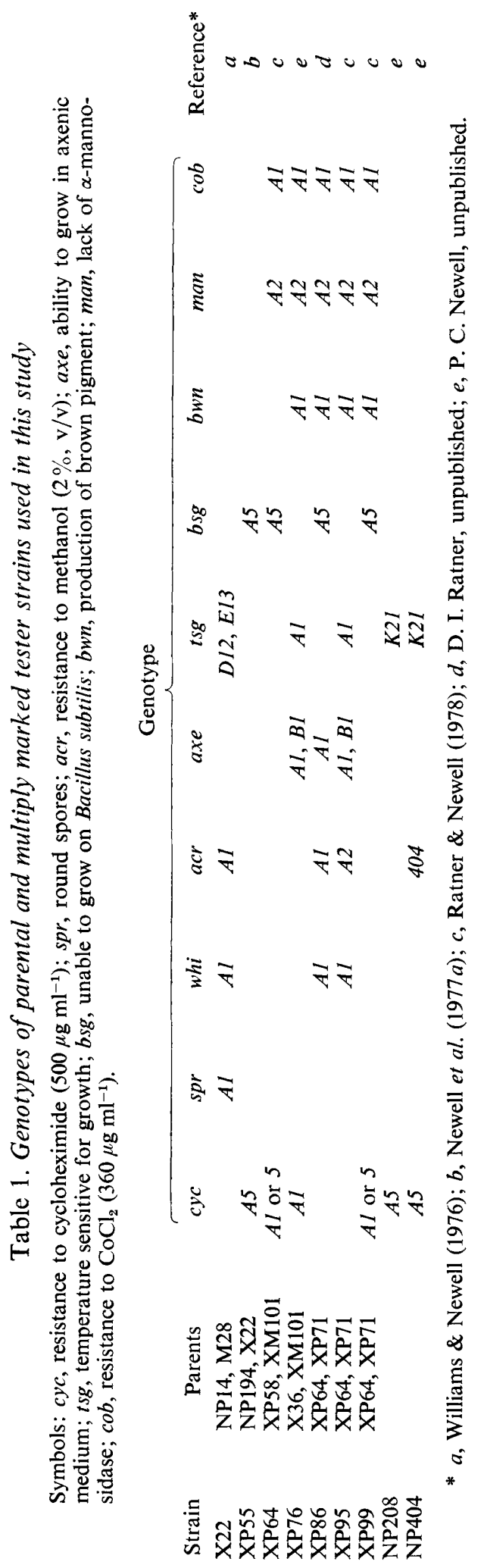




\section{RESULTS}

\section{Isolation of streamer mutants}

Using the parental $D$. discoideum strains XP55 and X22, 24 stable streamer mutants were isolated from 10 separate mutagenesis experiments. The streamer phenotype is the abnormal formation of large streams $(2.5$ to $20 \mathrm{~mm}$ in length) leading into the aggregation centre in clones on SM agar with $K$. aerogenes as bacterial associate. Under these conditions the streams of the parental strains are not normally discernible.

All the mutants grew and developed to form fruiting bodies with stalks containing normal spores. (Mutants bearing additional developmental mutations were counter-selected by the isolation procedure as this required all cells to pass through the spore stage for survival.) The size of the aggregates was characteristically different from mutant to mutant, but was invariably larger than those of the parental strains owing to the large territory size from which the amoebae collected. Such aggregates, however, were unable to produce correspondingly large fruiting bodies. The vast mass of cells seemed to be physically unwieldy and in most strains a large number of the cells could not enter the fruiting body but remained as a lump on the agar at the base of one to four short-stalked fruiting bodies that were constructed from each aggregate. In some strains (such as NP368 and NP375), however, the aggregates became completely subdivided thereby giving rise to several normal-sized fruiting bodies close together.

With some of the mutants that formed very large streams (such as NP377 and NP368) the streams appeared to be striped transversely. This phenomenon, which was easily observable with the naked eye in clones of these mutants, has been previously described in the 'Tiger' mutant (mutant M6.63) of Gross et al. (1977). Since the stripes moved slowly outward from the centre at a velocity of approximately $40 \mu \mathrm{m} \mathrm{min}^{-1}$, they were probably gross manifestations of the pulsatile signalling system.

\section{Complementation analysis}

Fusions with strains X22 and XP55. It was deduced that all the streamer mutations were recessive as diploids heterozygous for the streamer character $(\mathrm{stm} /+)$ were phenotypically wild type. It was therefore possible to carry out complementation tests between the different mutants.

Diploids formed from the fusion of mutants derived from strain X22 (bearing tsg mutations) with mutants derived from strain XP55 (bearing the bsg mutation) could be selected after the fusion procedure by plating amoebae on agar plates inoculated with B. subtilis and incubating for $4 \mathrm{~d}$ at $27^{\circ} \mathrm{C}$ (the non-permissive temperature) as described previously (Newell et al., 1977a). Using this technique, the three X22-derived mutants were found to be in different complementation groups and to show non-complementation with 13 of the XP55-derived mutants in three groups named $\operatorname{stm} A, \operatorname{stm} B$ and $\operatorname{stm} D$ (Table 2). The remaining eight XP55-derived mutants, however, complemented all three of the X22 mutants.

Replacement of $b s g A$ with $t s g A$. To select diploids formed from pairs of the remaining eight mutants, their $b s g$ mutations had first to be removed and be replaced by a $t s g$ mutation or (if this was impracticable) they had to be reverted. All eight mutants were consequently fused to form diploids with strain XP95 bearing $t s g A l$ in repulsion to the $b s g A 5$ marker on linkage group III. Stm Tsg Bsg ${ }^{+}$haploids were selected by segregation of the diploids on methanol- or cycloheximide-containing plates. Using such tsg derivatives in complementation tests with the original bsg-bearing strains, six of the eight mutants were shown to fall into two distinct complementation groups ( $\operatorname{stm} F$ and $\operatorname{stm} G$ ) and the remaining two mutants (NP383 and NP387), for which no tsg derivatives could be found, complemented both of these groups. 
Table 2. Complementation groups determined for the streamer mutants

The last two columns show derivative strains in which the $b s g A$ marker has been removed or reverted.

\begin{tabular}{|c|c|c|c|c|c|}
\hline $\begin{array}{l}\text { Com- } \\
\text { plementation } \\
\text { group }\end{array}$ & $\begin{array}{l}\text { Mutant } \\
\text { name }\end{array}$ & $\begin{array}{c}\text { Mutation } \\
\text { number }\end{array}$ & $\begin{array}{c}\text { Parental } \\
\text { strain }\end{array}$ & $\begin{array}{c}\text { Representative } \\
\text { Bsg }^{+} / \text {TsgA } \\
\text { derivatives }\end{array}$ & $\begin{array}{c}\text { Bsg }^{+} \\
\text {(revertants or } \\
\text { suppressors)/ } \\
\text { TsgK } \\
\text { derivatives }\end{array}$ \\
\hline stmA & $\begin{array}{l}\text { NP307 } \\
\text { NP294 } \\
\text { NP301 } \\
\text { NP378 } \\
\text { NP379 } \\
\text { NP382 } \\
\text { NP399 } \\
\text { NP400 }\end{array}$ & $\begin{array}{l}\text { stm-404 } \\
\text { stm-400 } \\
\text { stm-401 } \\
\text { stm-412 } \\
\text { stm-413 } \\
\text { stm-414 } \\
\text { stm-419 } \\
\text { stm-420 }\end{array}$ & $\begin{array}{l}\text { X22 } \\
\text { XP55 } \\
\text { XP55 } \\
\text { XP55 } \\
\text { XP55 } \\
\text { XP55 } \\
\text { XP55 } \\
\text { XP55 }\end{array}$ & XP162 & \\
\hline $\operatorname{stm} B$ & $\begin{array}{l}\text { NP306 } \\
\text { NP366 } \\
\text { NP371 } \\
\text { NP391 }\end{array}$ & $\begin{array}{l}\text { stm-403 } \\
\text { stm-405 } \\
\text { stm-408 } \\
\text { stm-417 }\end{array}$ & $\begin{array}{l}\text { X22 } \\
\text { XP55 } \\
\text { XP55 } \\
\text { XP55 }\end{array}$ & XP180 & \\
\hline $\operatorname{stm} C$ & NP383 & stm-415 & XP55 & & $\begin{array}{l}\text { XP174 } \\
\text { XP175 }\end{array}$ \\
\hline stmD & $\begin{array}{l}\text { NP304 } \\
\text { NP370 } \\
\text { NP372 } \\
\text { NP398 }\end{array}$ & $\begin{array}{l}\text { stm-402 } \\
\text { stm-407 } \\
\text { stm-409 } \\
\text { stm-418 }\end{array}$ & $\begin{array}{l}\text { X22 } \\
\text { XP55 } \\
\text { XP55 } \\
\text { XP55 }\end{array}$ & XP152 & \\
\hline stmE & NP387 & $s t m-416$ & XP55 & & $\begin{array}{l}\text { XP177 } \\
\text { XP178 }\end{array}$ \\
\hline$s t m F$ & $\begin{array}{l}\text { NP377 } \\
\text { NP401 }\end{array}$ & $\begin{array}{l}\text { stm-411 } \\
\text { stm- } 421\end{array}$ & $\begin{array}{l}\text { XP55 } \\
\text { XP55 }\end{array}$ & XP181 & \\
\hline$s t m G$ & $\begin{array}{l}\text { NP368 } \\
\text { NP375 } \\
\text { NP403 } \\
\text { NP411 }\end{array}$ & $\begin{array}{l}\text { stm-406 } \\
\text { stm-410 } \\
\text { stm-422 } \\
\text { stm-423 }\end{array}$ & $\begin{array}{l}\text { XP55 } \\
\text { XP55 } \\
\text { XP55 } \\
\text { XP55 }\end{array}$ & XP179 & \\
\hline
\end{tabular}

Reversion of bsg mutations. Mutants NP383 and NP387 could not be converted to $b_{s g^{+}}$ derivatives because all $\mathrm{bsg}^{+}$segregants were $\mathrm{Stm}^{+}$(due to their $\mathrm{stm}$ mutations being on linkage group III, see below). To overcome this problem it was decided to try isolating revertants (or suppressors) of the $b s g A 5$ mutation. However, in order that such a reverted or suppressed strain could be used for complementation testing, a tsg mutation had to be inserted into it prior to the $b s g$ reversion as without this it would be left without a diploid selector. The mutation $t s g K 21$ was used for this procedure as it was found to be an excellent diploid selector and was known to be unlinked to the $b s g A$ locus. Strain NP404 bearing tsgK21 was therefore fused to the two stm mutants NP383 and NP387 to yield tsg/+; +/bsg diploids, and Tsg Bsg Stm haploid segregants were selected from them by plating the diploids on methanol-containing plates. These haploids were then plated at high density $\left(3 \times 10^{6}\right.$ per plate) on to 15 plates of SM agar inoculated with a dense suspension of $B$. subtilis 36.1. After $7 \mathrm{~d}$ a total of two or three $\mathrm{Bsg}^{+}$clones were found growing from each haploid from the 15 plates. Whether these Stm Tsg Bsg ${ }^{+}$derivatives were true $b s g$ revertants or suppressors has not been determined, but all those investigated were capable of fusion at the normal frequency with other bsg-bearing strains. By fusion of these derivatives to NP383 and NP387 it was shown that the latter mutants bore stm mutations in different complementation groups, $\operatorname{stm} C$ and $\operatorname{stm} E$ (Table 2). 


\section{Linkage of streamer loci}

Segregation of diploids. Heterozygous diploids were made from all the stm mutants using the appropriate multiply marked tester strains (XP76 or XP99 for X22-derived mutants and XP95 for those derived from XP55; Ratner \& Newell, 1978). Haploids were then segregated from representative diploids formed from mutants in each of the complementation groups and tested for the pattern of segregation of markers (Table 3 ). In most cases the results revealed unambiguous linkage of the $\mathrm{stm}$ mutations to markers characteristic of particular linkage groups and lack of linkage to all the other groups.

However, with $\operatorname{stm} A$ and $\operatorname{stm} F$, problems were encountered with some mutations in clearly assigning them to a linkage group. Using diploids (such as DP828 and DP832) which were heterozygous for mutation $\operatorname{stmA404}$, the pattern of segregation of haploids clearly showed complete linkage of $\operatorname{stm} A$ to $\operatorname{cob} A$ on linkage group VII. Using diploids (such as DP817) heterozygous for $s t m A 400$, however, the pattern initially was difficult to interpret owing to the appearance of a number of non-streamers $\left(\mathrm{Stm}^{+}\right)$that were phenotypically $\mathrm{CobA}^{+}$non-parentals. These would not be expected if $\operatorname{stm} A$ and $\operatorname{cob} A$ were in repulsion on linkage group VII. Upon further subculture and retesting, this class was rescored as $\mathrm{Stm}^{+} \mathrm{CobA}$ (as shown in Table 3) and the pattern was thereby resolved. The problem was again encountered with diploids heterozygous for $\operatorname{stmF}$ (such as DP902 bearing $\operatorname{stmF411)}$, but in this case even after subculture not all $\mathrm{Stm}^{+} \mathrm{Cob}^{+}$segregants were reclassified (see Table 3).

To confirm the location of $\operatorname{stm} A$ on linkage group VII, diploids heterozygous for stm $A 400$ were plated on $\mathrm{CoCl}_{2}$-containing agar and the recombinant diploids $(\operatorname{cob} A / \operatorname{cob} A)$ that grew were selected for further study. (The haploids that also arose were discarded.) When several such independently isolated recombinant diploids were plated on methanolcontaining plates to select segregant haploids, no streamer haploids were isolated out of several hundred segregants tested. Controls with the original $\operatorname{cob} A / \operatorname{cob} A^{+}$diploids plated on methanol plates gave streamers as a very frequent segregant class. The simplest interpretation of such data is that when the cross-over event leading to $\operatorname{cob} A / \operatorname{cob} A$ homozygosity occurred the $\operatorname{stm} A /+$ locus was rendered homozygous $(+/+)$ by the recombination. This confirms that stm $A 400$ is located on linkage group VII and further indicates that stm $A$ is probably distal to the $c o b A$ marker.

Confirmation that $\operatorname{stm} A$ and $\operatorname{stm} F$ are on the same linkage group came from an experiment in which they were both placed in the same diploid. Although this diploid, which was $\operatorname{stm} A /+;+/ \operatorname{stm} F$, was a non-streamer due to complementation, all haploids that were subsequently segregated from it were found to be streamers, indicating that the two markers were in repulsion on the same linkage group in this diploid.

The tsgK marker. As an independent confirmation of the location of $\operatorname{stm} A$ and $\operatorname{stm} F$, use was made of the temperature sensitive for growth marker $t s g K$, as this had previously been found to show unambiguous linkage to $\operatorname{cob} A$ on linkage group VII. Representative segregations showing the location of $t s g K$ on linkage group VII are shown in Table 4. Diploids were constructed with $\operatorname{tsg} K$ in repulsion to $\operatorname{stm} A$ and to $\operatorname{stm} F$ and haploid segregants were selected on methanol-containing plates. The results (Table 4) clearly confirm that $\operatorname{stm} A$ and $\operatorname{stm} F$ are linked to the $t s g K^{+}$allele on linkage group VII.

Deviation from randomness. Although most stm loci could be readily located on definite linkage groups, the pattern of segregation seen was at times non-random. For example, all those XP55-derived streamer loci which are located on linkage group II (i.e. $\operatorname{stmB}, \operatorname{stm} D$ and $\operatorname{stm} G$ ) tended to produce only three classes of haploid progeny with reference to linkage group III. However, if haploids were selected on cycloheximide plates spread with $B$. subtilis, small numbers of the fourth class, i.e. $B$. subtilis-resistant streamers, were found. These all grew extremely poorly and it appears that there are unidentified mutations on linkage groups II and III which separately are not harmful but in combination are deleterious. 


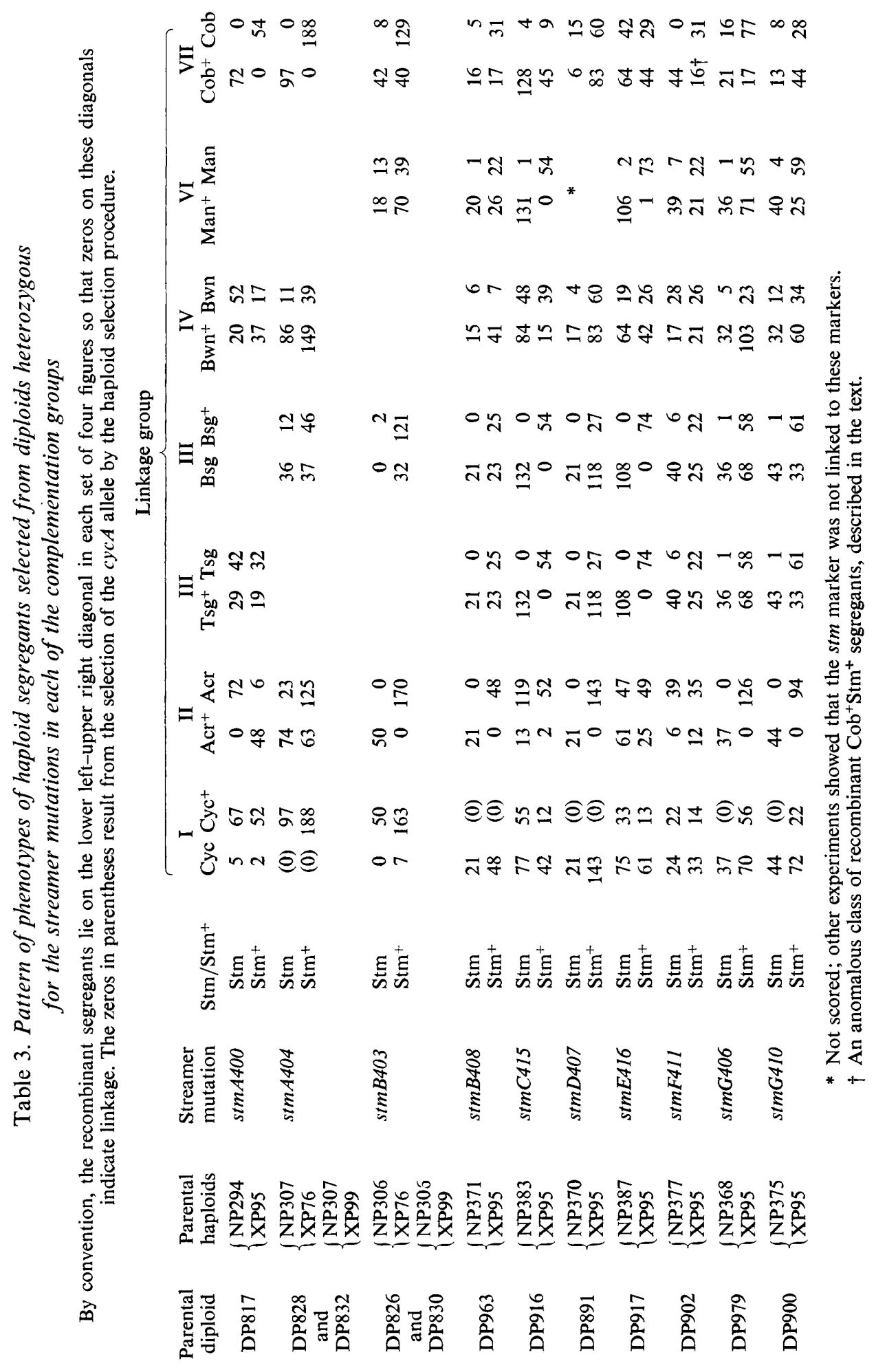




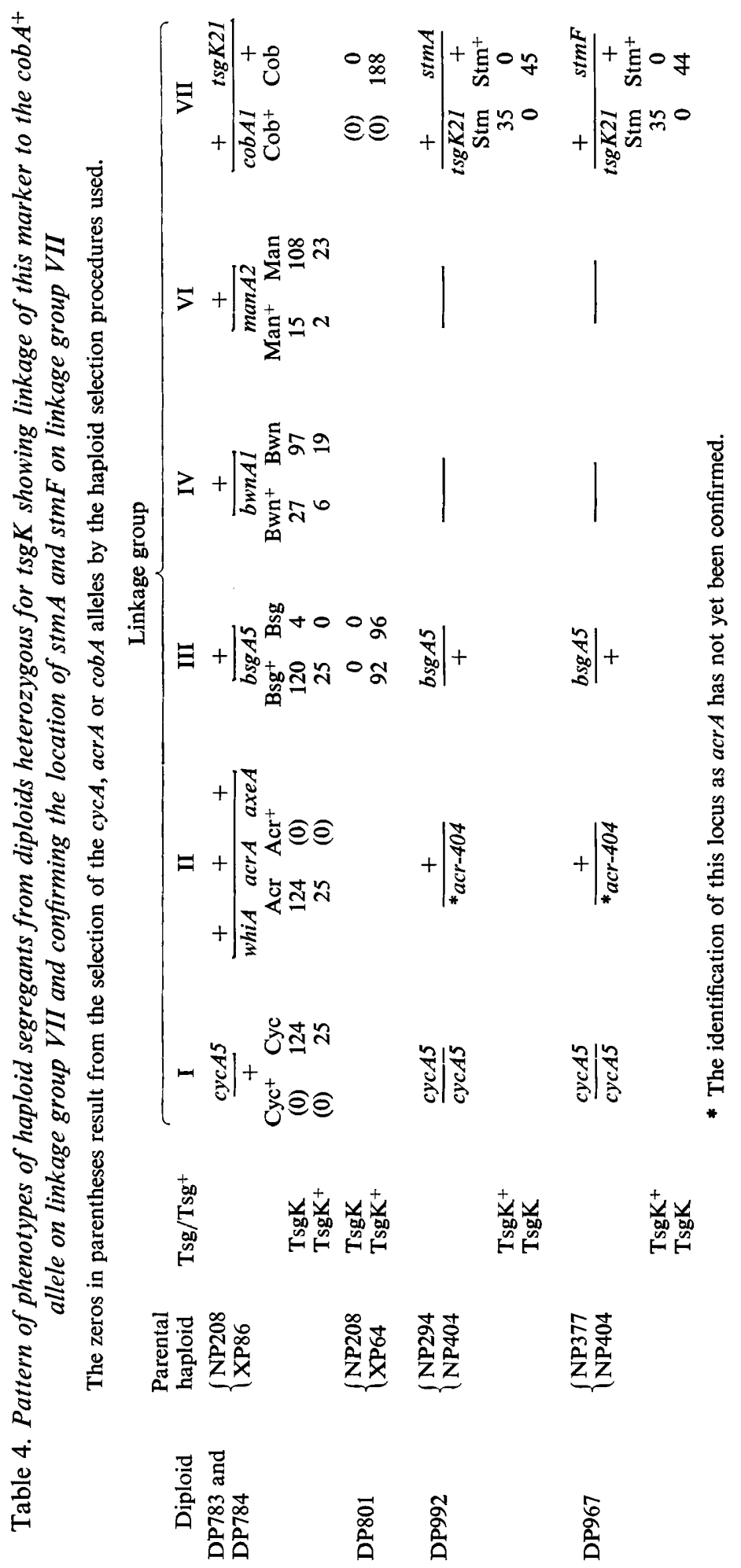




\section{Table 5. Assignment of complementation groups stmA to stmG to linkage groups}

The assignments are based on the patterns of segregation shown in Tables 3 and 4 and, in the case of $\operatorname{stm} A$, on mitotic recombination studies as described in the text.

$\begin{array}{ccc}\begin{array}{c}\text { Complementation } \\ \text { group }\end{array} & \begin{array}{c}\text { Linkage } \\ \text { group }\end{array} & \begin{array}{c}\text { Markers used } \\ \text { to identify linkage }\end{array} \\ \operatorname{stm} A & \text { VII } & \operatorname{cob} A, t \operatorname{sg} K \\ \operatorname{stm} B & \text { II } & a \operatorname{sr} A \\ \operatorname{stm} C & \text { III } & \operatorname{sig} A, t \operatorname{sg} A \\ \operatorname{stm} D & \text { II } & a \operatorname{si} A \\ \operatorname{stm} E & \text { III } & \operatorname{sig} A, t \operatorname{sg} A \\ \operatorname{stm} F & \text { VII } & \operatorname{cob} A, t \operatorname{sg} K \\ \operatorname{stm} G & \text { II } & a \operatorname{cr} A\end{array}$

There were also signs of non-randomness with $\operatorname{stm} C 415$ and stmE416. Both loci showed clear linkage to markers on linkage group III but they also showed such non-randomness with respect to linkage group VI that the exceptional classes needed careful retesting. This apparent 'near linkage' of groups III and VI was seen in all experiments involving diploids made from XP55 and XP95, but not in any other diploids. It was at first thought that a translocation involving linkage groups III and VI had taken place in one of these strains, probably XP55 as other workers had seen similar effects when using this strain (K. L. Williams, personal communication). However, small numbers of recombinant progeny for linkage group VI and the $\mathrm{stm}$ markers were always found. It therefore seems that the apparent association between these two linkage groups is something less substantial than a translocation and may be the manifestation of an incompatibility between unknown markers on these linkage groups. In order to confirm the location of stmC415 and stmE416 on linkage group III rather than VI, further experiments were performed in which only linkage groups III and VI were scored in larger numbers. The data for these experiments consistently showed no haploids recombinant for linkage group III while haploids recombinant for $\operatorname{stm} C$ and $\operatorname{stm} E$ and linkage group VI appeared at a frequency of between 1 and $3 \%$.

The linkage data is summarized in Table 5.

\section{DISCUSSION}

The streamer mutants belong to a class of aggregation pattern mutants with an easily recognizable phenotype that enables their isolation and genetic segregation to be readily undertaken. Within this class there are variations in the length of streams formed by the mutants and in the width of growth margins when clones are growing on plates inoculated with bacteria. Detailed comparison of the individual members of the seven complementation groups of streamer mutants showed that considerable variation in clonal phenotype occurs within the groups, particularly between mutants derived from different parental strains or the same mutations inserted into different genetic backgrounds. The streamer phenotype is clearly sensitive to the presence of unrelated minor mutations. The phenotypes shown in Fig. 1 are those most typical of each complementation group and are all of mutations in the parental strain XP55.

Linkage analysis of the mutants revealed that the seven complementation groups are located on groups II, III and VII. Use was made in this study of the multiply marked tester strains constructed in an earlier study (Ratner \& Newell, 1978) and these strains greatly facilitated the analysis by allowing a complete linkage test to be performed in one experiment. In a few experiments problems were encountered in scoring cobalt 


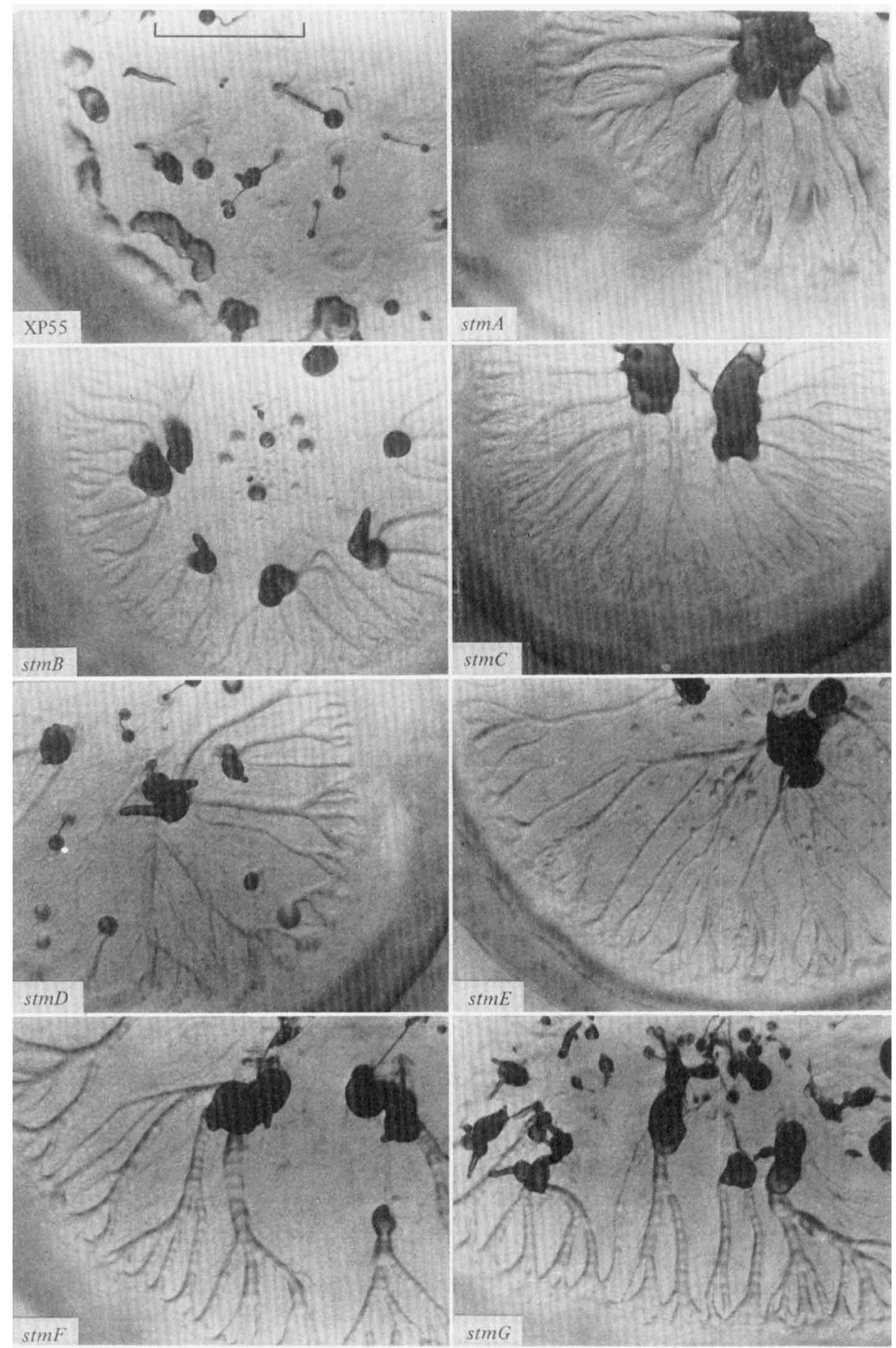

Fig. 1. Wild type XP55 with streamers representative of each complementation group: stmA, NP294; stmB, NP371; stmC, NP383; stmD, NP370; stmE, NP387; stmF, NP377; stmG, NP368. Bar marker represents $0.5 \mathrm{~cm}$. 
resistance (cobAl on linkage group VII) in segregants selected on methanol- or cycloheximide-containing plates. A class of false recombinant $\operatorname{cob} A^{+}$segregants was observed. This class disappeared in most experiments after retesting of the segregants, but it initially cast some doubt on the location of $\operatorname{stm} A$ and $\operatorname{stm} F$ on group VII. Independent evidence was sought using a new temperature sensitive for growth marker, $t s g K 21$, shown in separate experiments to be on this linkage group. This $t s g$ mutation gave unambiguous results in growth tests at the non-permissive temperature and has proved to be a valuable additional marker for linkage group VII.

All of the 24 streamer mutations isolated and used in this study can be placed in seven complementation groups. Two of the groups, however, contain only one member each (see Table 2) so it seems intuitively improbable that all complementation groups have been found. From the complementation data, 47 cases of non-complementation were observed or inferred for the complete cross of the 24 mutants with each other. Statistical analysis of such data using the method of frequency of allelism for a random distribution of mutations over the mutable loci (Lewontin \& Prout, 1956) indicates that the most likely number of loci that must be present to give the observed results is six (one less than the actual number found). The simplest explanation of such a result is that the observed distribution of $8,4,4,4,2,1,1$ of the mutations over the seven complementation groups is non-random and it seems most likely that it is the $\operatorname{stm} A$ class with eight mutants that is unbalancing the distribution. If this locus is assumed to represent a mutational 'hot-spot' that is over-represented, then the calculation would suggest an upper estimate of nine for the number of loci able to give the streamer phenotype. Although due care must be taken in the interpretation of such numbers it does seem likely that most of the streamer loci are represented in this genetic study.

A study by Riedel et al. (1973) suggested that large aggregation territory size of mutants might be correlated with a deficiency of the enzyme phosphodiesterase which destroys the cyclic AMP used for signalling by $D$. discoideum. Preliminary data from our streamer mutants, however, suggest that such a correlation cannot be made for these mutants. Further biochemical investigations of representatives of the seven known complementation groups is now in progress.

We wish to thank Stephanie Rogers for excellent technical assistance, David Ratner for helpful discussions, Frank Caddick for photographic developing and printing and Dorota Mosses for the isolation in this laboratory of mutants NP294, NP301, NP304, NP306 and NP307. Fiona M. Ross is the recipient of an M.R.C. studentship.

\section{REFERENCES}

Coukell, M. B. \& Roxby, N. M. (1977). Linkage analysis of developmental mutations in aggregation-deficient mutants of Dictyostelium discoideum. Molecular and General Genetics 151, 275-288.

Demerec, M., Adelberg, E. A., Clark, A. J. \& Hartman, P. E. (1966). A proposal for a uniform nomenclature in bacterial genetics. Genetics $\mathbf{5 4}$, 61-76.

Francis, D. (1977). Synthesis of developmental proteins in morphogenetic mutants of Polysphondylium pallidum. Developmental Biology 55, 339-346.

Green, A. A. \& Newell, P. C. (1975). Evidence for the existence of two types of cAMP binding sites in aggregating cells of Dictyostelium discoideum. Cell 6, 129-136.
Gross, J., Kay, R., Lax, A., Peacey, M., Town, C. \& Trevan, D. (1977). Cell contact, signalling and gene expression in Dictyostelium discoideum. In Development and Differentiation in the Cellular Slime Moulds, pp. 135-147. Edited by P. Cappuccinelli \& J. Ashworth. Amsterdam: Elsevier/ North Holland.

Kessin, R. H., Williams, K. L. \& Newell, P. C. (1974). Linkage analysis in Dictyostelium discoideum using temperature-sensitive growth $\mathrm{mu}$ tants selected with bromodeoxyuridine. Journal of Bacteriology 119, 776-783.

Lewontin, R. C. \& Prout, T. (1956). Estimation of the number of different classes in a population. Biometrics 12, 211-223.

Loomis, W. F. (1975). Dictyostelium discoideum: $a$ Developmental System. New York: Academic Press. 
Loomis, W. F., White, S. \& Dimond, R. L. (1976). Sequence of dependent stages in Dictyostelium discoideum. Developmental Biology 53, 171-177.

MANDElstam, J. (1969). Regulation of bacterial spore formation. Symposia of the Society for Experimental Biology 19, 337-402.

Mosses, D., Williams, K. L. \& Newell, P. C. (1975). The use of mitotic crossing-over for genetic analysis in Dictyostelium discoideum: mapping of linkage group II. Journal of General Microbiology 90, 247-259.

Newell, P. C. (1977). Aggregation and cell surface receptors in cellular slime molds. In Receptors and Recognition, Series B, vol. 3, pp. 1-57. Edited by J. L. Reissig. Chapman \& Hall.

NEWELL, P. C. (1978). Genetics of the cellular slime moulds. Annual Review of Genetics 12, 69-93.

Newell, P. C., Henderson, R. F., Mosses, D. \& RATNER, D. I. (1977a). Sensitivity to Bacillus subtilis: a novel system for selection of heterozygous diploids of Dictyostelium discoideum. Journal of General Microbiology 100, 207-211.

Newell, P. C., Ratner, D. I. \& Wright, M. D. $(1977 b)$. New techniques for cell fusion and linkage analysis of Dictyostelium discoideum. In Development and Differentiation in the Cellular Slime Moulds, pp. 51-61. Edited by P. Cappuccinelli \& J. Ashworth. Amsterdam: Elsevier/ North Holland.

RAPER, K. B. (1935). Dictyostelium discoideum, a new species of slime mold from decaying forest leaves. Journal of Agricultural Research 50, 135147.

Ratner, D. I. \& Newell, P. C. (1978). Linkage analysis in Dictyostelium discoideum using multiply marked tester strains: establishment of linkage group VII and the reassessment of earlier linkage data. Journal of General Microbiology 109, 225236.

Riedel, V., Gerisch, G., Muller, E. \& Beug, H. (1973). Defective cyclic adenosine- $3^{\prime}, 5^{\prime}$-phosphatephosphodiesterase regulation in morphogenetic mutants of Dictyostelium discoideum. Journal of Molecular Biology 74, 573-585.

Williams, K. L., Kessin, R. H. \& Newell, P. C. (1974). Parasexual genetics in Dictyostelium discoideum: mitotic analysis of acriflavin resistance and growth in axenic medium. Journal of General Microbiology 84, 59-69.

Williams, K. L. \& Newell, P. C. (1976). A genetic study of aggregation in the cellular slime mould Dictyostelium discoideum using complementation analysis. Genetics 82, 287-307.

ZadA-HAMes, I. M. \& AshwORTH, J. M. (1977). The cell cycle during the growth and development of Dictyostelium discoideum. In Development and Differentiation in the Cellular Slime Moulds, pp. 69-78. Edited by P. Cappuccinelli \& J. Ashworth. Amsterdam: Elsevier/North Holland. 